\title{
miR-146a in Cardiovascular Diseases and Sepsis: An Additional Burden in the Inflammatory Balance?
}

\author{
Ana B. Arroyo ${ }^{1, *}$ Sonia Águila ${ }^{1, *}$ María P. Fernández-Pérez ${ }^{1} \quad$ Ascensión M. de los Reyes-García $^{1}$ \\ Laura Reguilón-Gallego ${ }^{1}$ Laura Zapata-Martínez ${ }^{1}$ Vicente Vicente ${ }^{1}$ Constantino Martínez ${ }^{1, * *}$ \\ Rocío González-Conejero ${ }^{1, * *}$
}

${ }^{1}$ Department of Hematology and Medical Oncology, Morales Meseguer University Hospital, Centro Regional de Hemodonación, Universidad de Murcia, IMIB, Murcia, Spain

Thromb Haemost 2021;121:1138-1150.
Address for correspondence Rocío González-Conejero, PhD, Centro Regional de Hemodonación, C/ Ronda de Garay S/N, 30003 Murcia, Spain (e-mail: rocio.gonzalez@carm.es).

Constantino Martinez, PhD, Centro Regional de Hemodonación, Cl Ronda de Garay S/N, 30003 Murcia, Spain (e-mail: constant@um.es).

\begin{abstract}
Keywords

- polymorphisms

- miRNA

- cardiovascular disease

The new concept of thrombosis associated with an inflammatory process is called thromboinflammation. Indeed, both thrombosis and inflammation interplay one with the other in a feed forward manner amplifying the whole process. This pathological reaction in response to a wide variety of sterile or non-sterile stimuli eventually causes acute organ damage. In this context, neutrophils, mainly involved in eliminating pathogens as an early barrier to infection, form neutrophil extracellular traps (NETs) that are antimicrobial structures responsible of deleterious side effects such as thrombotic complications. Although NETosis mechanisms are being unraveled, there are still many regulatory elements that have to be discovered. Micro-ribonucleic acids (miRNAs) are important modulators of gene expression implicated in human pathophysiology almost two decades ago. Among the different miRNAs implicated in inflammation, miR-146a is of special interest because: (1) it regulates among others, Toll-like receptors/nuclear factor-kB axis which is of paramount importance in inflammatory processes, (2) it regulates the formation of NETs by modifying their aging phenotype, and (3) it has expression levels that may decrease among individuals up to $50 \%$, controlled in part by the presence of several polymorphisms. In this article, we will review the main characteristics of miR-146a biology. In addition, we will detail how miR$146 \mathrm{a}$ is implicated in the development of two paradigmatic diseases in which thrombosis and inflammation interact, cardiovascular diseases and sepsis, and their association with the presence of miR-146a polymorphisms and the use of miR-146a as a marker of cardiovascular diseases and sepsis.
\end{abstract}

\section{Introduction}

Thromboinflammation relies on the interplay between thrombosis and inflammation (through the activation of the hemostatic and coagulation system and the innate and

\footnotetext{
* Equally contributed.

** Shared senior authorship.
}

\section{received}

September 13, 2020

accepted after revision

December 18, 2020

published online

December 22, 2020 adaptive immunity) and it has been recognized as a pathophysiological process in response to a wide variety of sterile and non-sterile stimuli causing in some cases acute organ damage. ${ }^{1,2}$ Of special interest in this field are micro-ribonucleic acids (miRNAs), and among them, we here highlight miR-146a. This miRNA has been extensively studied given its prominent regulatory role in inflammatory and immune processes. ${ }^{3,4}$ Additionally, genetic and epigenetic regulation

$\begin{array}{ll}\text { @ } 2020 . \text { Thieme. All rights reserved. } & \text { DOI https://doi.org/ } \\ \text { Georg Thieme Verlag KG, } & \text { 10.1055/a-1342-3648. } \\ \text { Rüdigerstraße 14, } & \text { ISSN 0340-6245. }\end{array}$

70469 Stuttgart, Germany 
of miR-146a expression in humans provide excellent models to evaluate the role of this miRNA in different pathologies. ${ }^{5-7}$

In this review, we will summarize the biology of miR-146a and its function in the interplay between inflammation and thrombosis, especially focusing in cardiovascular diseases (CVDs) and sepsis. These pathologies have been over the years separated in different categories. Interestingly, it has recently been proposed that they both share pathophysiological signaling pathways and common genetic variants. ${ }^{8}$ Thus, both diseases share similar endpoints of inflammation, coagulation, and endothelial activation..$^{9,10}$

\section{Biosynthesis of miRNAs}

It has been almost two decades since miRNAs were discovered as important regulators of gene expression in disease. ${ }^{11}$ miRNA maturation is a complex process that starts in the nucleus where miRNA genes are transcribed by RNA polymerase II, giving rise to a product called pri-miRNA that is further processed into pre-miRNA by the microprocessor catalytic complex, composed by DROSHA and DGCR8. The resulting pre-miRNA molecule is a 3'overhang hairpin-like structure of approximately 60 nucleotides. The pre-miRNA is then exported to the cytoplasm by a RanGTP-dependent exportin (XPO5) allowing an additional processing step by DICER resulting in a new approximately 22 nucleotide mature double-strand miRNA. ${ }^{12}$ At that point, a single-stranded miRNA is inserted in a functional macromolecular unit, containing among other the argonaute protein 2 (AGO2), recognizing its target messenger RNA ( $\mathrm{mRNA}$ ) by base pairing, mainly in the 3' untranslated region, and allowing the inhibition of mRNA translation and/or the mRNA decay. ${ }^{13}$ To date, 2,654 mature miRNAs (Mirbase, Release 22.1: October 2018) have been described in humans, that may repress the majority of the transcriptome, regulating different physiological and pathological processes. ${ }^{14}$

\section{miR-146a Genetics and Regulation}

The gene encoding mature miR-146a (MIR146A) is located on chromosome 5 ( 5 q33.3) in humans and on chromosome 11 (B1.1) in mice. As it happens for the majority of miRNAs, miR-146a is highly conserved in mammalian (www.mirbase. org). The mature sequences were first assigned the name of the miRNA with or without an asterisk, that is, miR-146a (the predominant product) and miR-146a* (from the opposite arm of the precursor), but later (2011) a definitive nomenclature, miR-146a-5p (from the $5^{\prime}$ arm) and miR-146a-3p (from the $3^{\prime}$ arm), was established. In the case of miR-146a, the mature form that has been more extensively studied is miR-146a-5p. Since our studies and the references included in the review refer to this form, we used miR-146a throughout the text. Indeed, only a few studies have analyzed the role of miR-146a-3p and they were not included in the present review. Additionally, letter suffix denotes closely related mature sequences, in the case of miR-146a, there is a close miRNA, miR-146b (located in chromosome 10) that differs from miR-146a by two nucleotides located in the 3' end, both
miRNAs share the same seed region (located in the $5^{\prime}$ end) and most of the targets. The regulation of miR-146a expression is complex and may be performed at different levels as we will describe below. miR-146a transcription is executed by a promoter located $16 \mathrm{~kb}$ upstream of MIR146A. ${ }^{15}$ Different putative binding sites for a series of transcription factors were characterized in this promoter (-Fig. 1A). Taganov et al demonstrated that miR-146a expression was mainly regulated by nuclear factor- $\mathrm{KB}(\mathrm{NF}-\mathrm{KB})$ through Toll-like receptors (TLRs) such as TLR4 that is activated by lipopolysaccharide (LPS). In addition, other cytokines such as interleukin-1 $\beta$ (IL-1 $\beta$ ) or tumor necrosis factor (TNF- $\alpha$ ) also increased the levels of mature miR-146a. ${ }^{15}$ Another study revealed that the transcription factor ETS- 1 could potentially regulate the expression of miR-146a. ${ }^{16}$ Indeed, an ETS- 1 knockdown model provokes the inability to induce miR-146a expression in vitro. ${ }^{17}$ On the opposite side, in vitro models showed that a c-Myc binding site, located in the promoter region, is able to repress the expression of miR-146a. ${ }^{18}$ But other regulatory processes also take place at a genetic level. Different singlenucleotide polymorphisms (miR-SNPs) have been described to modulate miR-146a levels. ${ }^{17}$ In this review, we will focus on three, rs2431697, rs2910164, and rs57095329, that have been extensively studied ( $\mathbf{- F i g . 1 A}$ ). The first functional miR-SNP to be characterized was rs57095329 (MAF 0.26 in Asians and 0.025 in Europeans; https://www.ncbi.nlm.nih.gov/snp/). ${ }^{17}$ This miR-SNP is located in the miR-146a promoter and the G allele decreases the binding affinity of ETS-1, reducing miR146a levels by approximately $40 \%{ }^{17}$ Another widely studied miR-SNP is rs2910164 (MAF 0.70 in Asians and 0.24 in Europeans; https://www.ncbi.nlm.nih.gov/snp/) that is located in the pre-miR-146a. The minor C allele causes mispairing within the hairpin affecting the efficiency of pri-miR-146a processing and probably the stability and/or efficiency of pre-miR-146a export to the cytoplasm ${ }^{19}$ and reducing the levels by more than $40 \%$ in CC homozygous. ${ }^{20}$ Rs2910164 has been associated with various diseases where inflammation is an important issue. ${ }^{7,21,22}$ Finally, rs2431697 is another miR-SNP located approximately $30 \mathrm{~kb}$ upstream of pre-miR-146a. Löfgren et $\mathrm{al}^{20}$ showed that the presence of the minor $\mathrm{T}$ allele reduces both pri-miR-146a levels as well as mature miR-146a levels ( $\sim 50 \%)$. The mechanism is not well defined and other miR-SNPs in linkage disequilibrium may be involved. $^{20}$ We will thoroughly develop the effects of rs2431697 together with rs2910164 in thrombosis in pathologies with inflammatory background in the next paragraphs.

Other factors such as long non-coding (lnc)-RNAs are newly described regulators of miR-146a. These molecules can modulate miR-146a expression preferentially by a sponging mechanism (-Fig. 1B). Thus, metastasis-associated lung adenocarcinoma transcript 1 (MALAT1) knockdown lead to reduction of phosphorylated inhibitor of NF-KB in a rat model of LPS-induced acute kidney injury ${ }^{23}$ and suppressed inflammatory response by upregulating miR-146a in LPS-induced acute lung injury. ${ }^{24}$ LncRNA $X$ inactivate-specific transcript (XIST), in turn, by sponging miR-146a, diminished the mechanical pain threshold due to the upregulation of voltage-gated sodium channel $1.7\left(\mathrm{Na}_{\mathrm{v}} 1.7\right)$ in an animal pain model. ${ }^{25}$ Finally, 

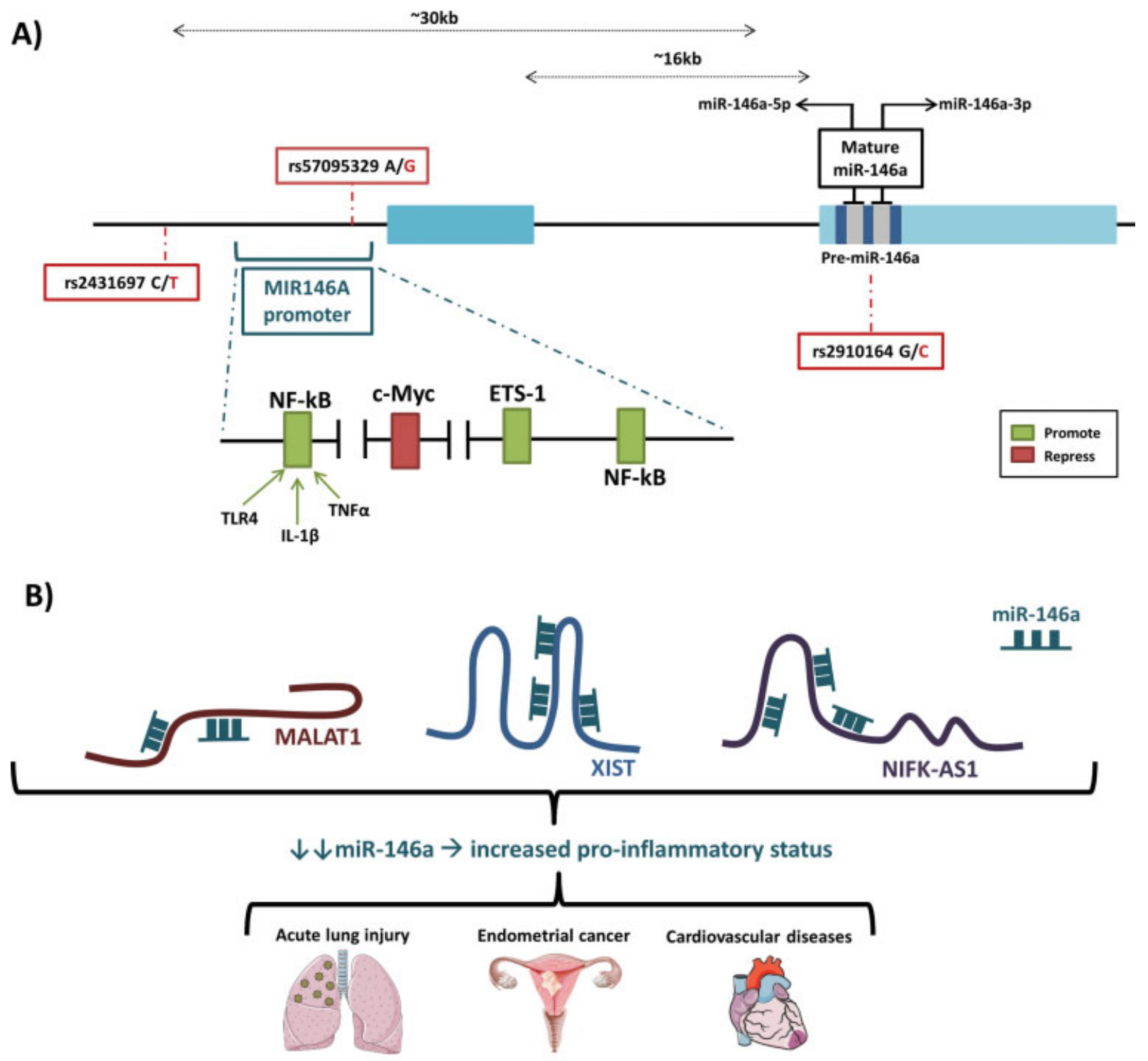

Fig. 1 miR-146a location and regulation. (A) miR-146a encoding gene is located in the human chromosome 5 (5q33.3). miR-146a transcription depends of a promoter located $\sim 16 \mathrm{~kb}$ upstream of the MIR146A gene. Putative binding sites for transcription factors are shown as well as the location of the miR-single-nucleotide polymorphisms (SNPs) than can modulate miR-146a levels. (B) miR-146a may also be modulated by several long non-coding ribonucleic acids (Lnc-RNAs), generally by a sponging mechanism, this may lead to a higher inflammatory status that may be derived in the aggravation of certain pathologies.

in endometrial cancer, NIFK antisense RNA 1 (NIFK-AS1) inhibited the M2-like polarization of macrophages reducing the estrogen-induced proliferation, migration, and invasion of cancer cells by reducing miR-146a-5p levels through a sponging mechanism. ${ }^{26}$

\section{Biological Functions of miR-146a in Immune Cells}

miR-146a is a critical molecular brake of inflammation that regulates among other the TLR4/NF-KB pathway. Indeed, Taganov et al demonstrated in THP-1 cells that miR-146a directly modulates the expression of TNF receptor-associated factor 6 (TRAF6) and IL-1 receptor-associated kinase 1 (IRAK1) that play an essential role in controlling the TLR4/NF-KB pathway ${ }^{15}$ (-Fig. 2). A few in vitro studies have shown that miR-146a may also regulate the expression of TLR4, but in vivo studies are mandatory to further confirm this important regulation. ${ }^{27-29}$ As mentioned above, LPS through TLR4/NF-KB promotes the expression of inflammatory cytokines such as IL-6, IL-8, IL-1 $\beta$, or TNF- $\alpha$ and miR-146a controls overwhelmed cellular response to inflammatory signals through a negative feedback regulatory loop. ${ }^{15,30,31}$ In 2011, Baltimore's laboratory published two studies using a deficient mouse model for miR-146a that gave many clues on the pathological role of miR-146a. ${ }^{30,32}$ These mice react to LPS challenge with an important inflammatory response displaying high levels of IL-6 in serum, among other. Interestingly, aging also provokes them an increased inflammatory status. Indeed, the authors showed that aged $m i R-146 a^{-1-}$ mice developed a myeloproliferative phenotype and tumors in their secondary lymphoid organs. ${ }^{30}$ These data suggest a role for miR-146a far beyond the 


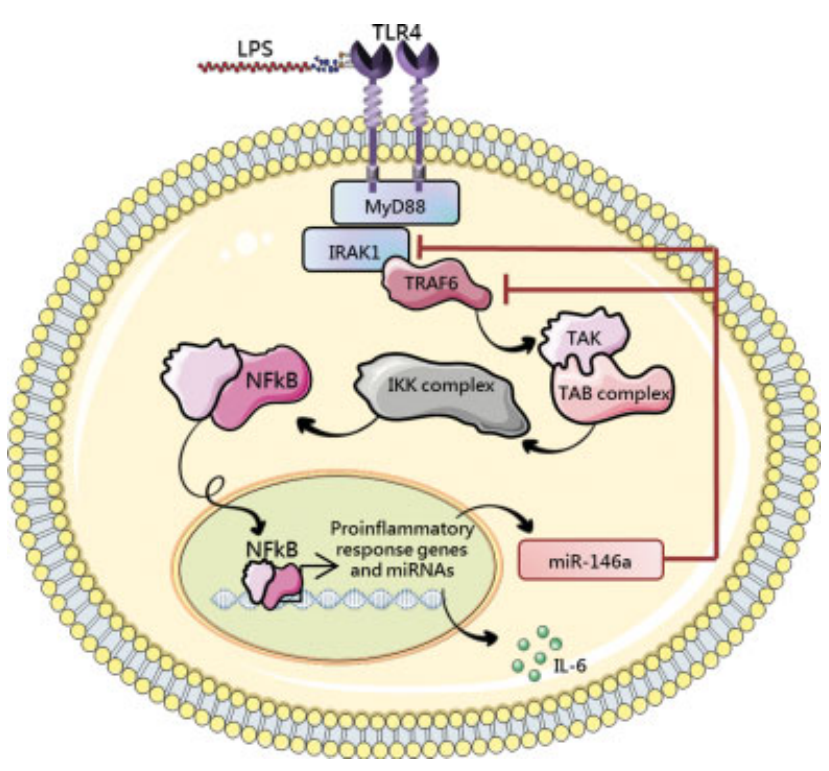

Fig. 2 Regulation of the Toll-like receptor (TLR) 4/nuclear factor-kB (NF-kB) axis by miR-146a. Binding of lipopolysaccharide (LPS) to TLR4 leads to the activation of NF-kB and its translocation to the nucleus. NF-kB induces the transcription of several proinflammatory genes (such as interleukin [IL]-6) and also promotes the transcription of pri-miR-146a that after a series of maturation processes gives rise to the mature miR-146a that will act through a negative feedback regulatory loop to essentially control the levels of TRAF6 and IRAK1, main players of the TLR4/NF-kB axis.

mere response to an endotoxin challenge since the deficiency of this miRNA constitutively affects NF-KB signaling. ${ }^{30,32}$ In this line, our group recently demonstrated that rs2431697 TT genotype is an early predictor of myelofibrosis progression, independent of the JAK2V617F allele burden. ${ }^{33}$ Janus kinase (JAK)/signal transducer and activator of transcription (STAT) signaling pathway plays a critical role in myeloproliferative neoplasms pathogenesis by driving both the malignant clone and the inflammatory microenvironment. Thus, JAK2V617F allele burden is one of the main drivers of clonal expansion toward end-stage disease. ${ }^{33}$ Our results showed that rs2431697 genotype increases Jak/Stat signaling, as we demonstrated in $m i R-146 a^{-1-}$ mice, probably due to the elevation of systemic IL-6 levels. ${ }^{33,34}$

One of the crucial elements implicated in thrombosislinked inflammation is the neutrophil. These cells are mainly involved in eliminating pathogens and being an early barrier to infection. ${ }^{35}$ In 2004, Brinkmann et al, in a landmark study, characterized a new mechanism, later termed NETosis, by which neutrophils are able to remove bacteria under certain stresses such as infection. ${ }^{36}$ NETs are structures composed of nuclear chromatin and associated with nuclear histones as well as cytoplasmic and granular antimicrobial proteins. ${ }^{37}$ Their association with CVDs, venous thrombosis, and autoimmune diseases has been largely documented. ${ }^{37-39}$ We recently showed that miR-146a was involved in NET formation using a miR-146a ${ }^{-1-}$ mouse model. Our results demonstrated that miR-146a deficiency promotes in vitro the intrinsic capacity of neutrophils to form NETs in response to phorbol 12-myristate 13 -acetate. ${ }^{40}$ On the other hand, sterile stresses such as atherosclerosis and non-sterile such as endotoxemia produced an increased in NETosis in $m i R-146 a^{-1-}$ mice versus wild-type
(WT) ${ }^{40}$ Although the precise mechanism is unknown, the lack of miR-146a provoked changes in neutrophils that display an aging phenotype characterized by the markers CD62 $\mathrm{L}^{\text {low }}$ CD11 $b^{\text {high }}$ Cxcr $4^{\text {high }}$, and an overexpression of Tlr4 in the aged population. ${ }^{41}$ miR-146a ${ }^{-1-}$ neutrophils also showed a lower expression of $\mathrm{Cxcr} 1$, that has been associated with a proinflammatory phenotype. All these features together with an increased formation of reactive oxygen species observed in miR-146a ${ }^{-1-}$ mice, by a yet to discover mechanism, may explain in part why miR-146a deficiency increases NETosis. ${ }^{41}$

In addition, miR-146a has a key role in macrophages polarization and tolerance, relevant processes in atherosclerosis development. ${ }^{42}$ Importantly, miR-146a deficiency promoted M1 phenotype and its overexpression resulted in M2 macrophages by decreasing the expression of NOTCH receptor 1 (NOTCH1) an important regulator of macrophage differentiation and activation ( $\mathbf{F i g}$. 3A). ${ }^{43,44}$ This miRNA also inhibited the differentiation to M1 of hepatic macrophages by targeting signal transducer and activator of transcription 1 (STAT1) and consequently the interferon- $\gamma$ signaling. ${ }^{45}$

Oxidized low-density lipoprotein (oxLDL), an important inducer of atherosclerosis, may also regulate miR-146a expression. OxLDL-stimulated macrophages presented a
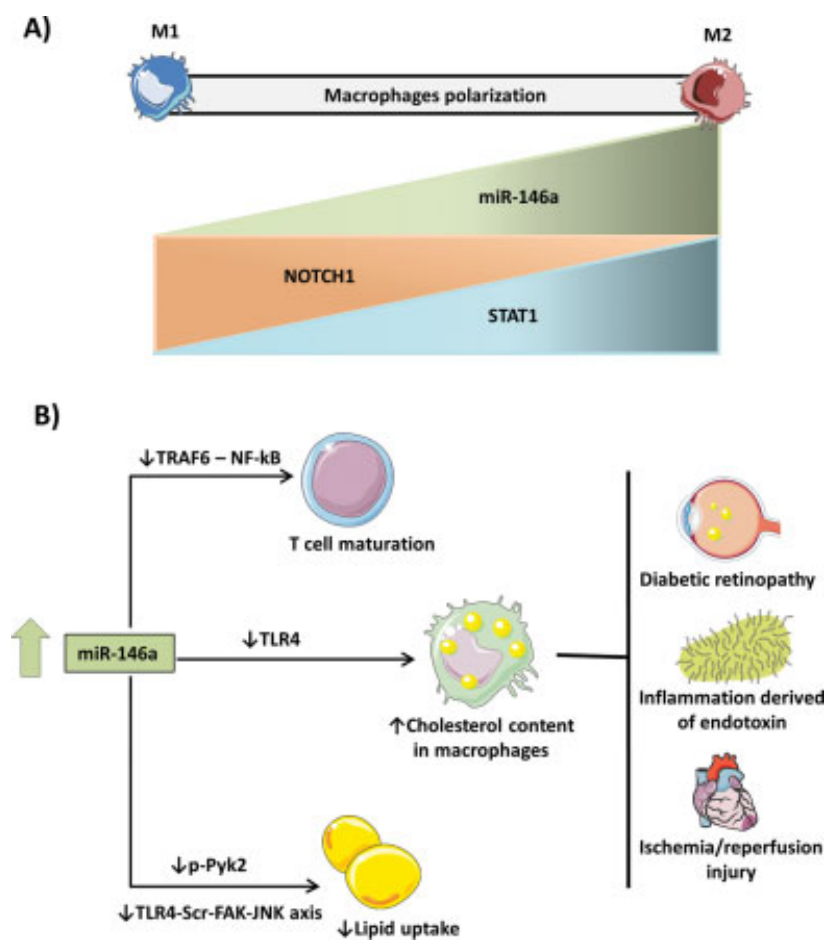

Fig. 3. Role of miR-146a on atherosclerotic inflammatory components. (A) miR-146a deficiency promotes macrophage polarization to M1 phenotype. Conversely, miR-146a overexpression promotes $\mathrm{M} 2$ phenotype by decreasing the expression of NOTCH1 and inhibited the differentiation to M1 by targeting STAT1. (B) miR-146a overexpression can block the TLR4-SrcFAK-JNK axis and inhibit the Pyk2 phosphorylation leading to the inhibition of lipid uptake. This overexpression can also decrease the nuclear factor-kB (NF-KB) activity through repressing TRAF6, resulting in T cells maturation. By provoking a lower TLR4 expression and higher intake of cholesterol by macrophages, the high levels of miR-146a may finally lead to diabetic retinopathy, inflammation derived of endotoxin, or ischemia/reperfusion injury, among other pathologies. 
downregulation of miR-146a through lectin-type oxLDL receptor 1 (LOX-1). ${ }^{46}$ In contrast, miR-146a overexpression provoked a reduction in TLR4 expression and higher cholesterol content in macrophages. ${ }^{28}$ miR-146a may inhibit lipid uptake by blockade of TLR4-Src-FAK-JNK axis and the phosphorylation of protein tyrosine kinase $2 \beta$ (Pyk2) and paxillin, a signal transduction adaptor protein. ${ }^{28}$ Accordingly, the expression of IL-6, IL-8, monocyte chemoattractant protein-1, and matrix metallopeptidase 9 decreased via miR-146a via TLR4-TRAF6/IRAK1-AKT. ${ }^{46}$ Indeed, the direct repression of TLR4 by miR-146a has been extensively characterized in different pathologies such as diabetic retinopathy, inflammation derived of endotoxin, and ischemia/reperfusion (I/R) injury (- Fig. 3B)..$^{29,47,48}$ On the other hand, miR-146a has also a role in adaptive immunity regulating $\mathrm{T}$ cell maturation and response against acute or chronic inflammation in a TRAF6-NF-KB-dependent manner. ${ }^{49,50}$

\section{Relevance of miR-146a in the Pathophysiology Underlying Thromboinflammatory Diseases}

\section{Atherosclerosis}

Atherosclerosis is the main underlying process driving to most cardiovascular pathologies including coronary artery disease (CAD), ischemic stroke (IS), or acute myocardial infarction (AMI). ${ }^{51}$ Progressing atherosclerotic plaques may eventually rupture, thereby inducing intraluminal thrombosis leading to adverse cardiac events. Since inflammation is a key contributor to all stages of atherosclerosis and its fatal cardiovascular consequences, miR-146a was assumed to be a promising anti-inflammatory and atheroprotective agent. Nonetheless, apolipoprotein $\mathrm{E}(\mathrm{ApoE})$ controls inflammation by suppressing NF-KB signaling and protects from atherosclerosis and inflammatory diseases. ${ }^{51} \mathrm{Li}$ et $\mathrm{al}^{52}$ reported that ApoE increased the expression of transcription factor PU.1 raising the levels of miR146a in monocytes/macrophages and repressing the NF- $\mathrm{KB}$ signaling. Importantly, systemic intravascular delivery of miR146a attenuated macrophage activation, atherosclerosis, and proinflammatory response in both, ApoE ${ }^{-1-}$ and $\mathrm{Ldlr}^{-1-}$ hyperlipidemia mouse models. ${ }^{52}$ These findings established for the first time that enhancing miR-146a expression could antagonize atherogenesis. Intriguingly, we observed that lack of miR-146a exclusively in the hematopoietic compartment did not affect atherosclerotic plaque formation in $\mathrm{Ldlr}^{-1-}$ mice at early and late stages of disease progression. ${ }^{53}$ Similarly, Cheng et $\mathrm{al}^{54}$ showed no differences in atherosclerotic burden in $L d l r^{-1-}$ mice transplanted with miR-146a $a^{-1-}$ bone marrow (BM) at early stages of disease despite having elevated levels of circulating proinflammatory cytokines. However, these authors found that miR-146a ${ }^{-1-}$ mice receiving WT BM transplantation had enhanced endothelial cell activation and elevated atherosclerotic plaque burden compared with $\mathrm{Ldlr}^{-1-}$ mice receiving WT BM, demonstrating the atheroprotective role of miR-146a in the endothelium..$^{54}$ In concordance with these results, it has been reported that endothelium-specific delivery of miR-146a-loaded E-selectin-targeting microparticles decreased plaque size and macrophage infiltration in $A p o E^{-l-}$ mice. ${ }^{55}$ Thus, atheroprotection upon systemic
miR-146a administration may therefore be caused by specific effects on vascular cells. In previous studies, the inhibition of NF-KB activation in endothelial cells reduced atherogenesis, ${ }^{56}$ whereas inhibition of NF-KB activation in macrophages resulted in enhanced atherosclerosis in $\mathrm{Ldlr}^{-I-}$ mice. ${ }^{57}$ Additionally, miR-146a was enriched in extracellular vesicles (EVs) from mouse and human macrophages treated with an atherogenic stimulus (oxLDL), demonstrating an EV-mediated delivery of miR-146a that repressed target genes involved in cell migration and adhesion pathways in recipient cells. ${ }^{58}$ Moreover, miR-146a levels were paradoxically elevated in plaques from atherosclerotic mice. ${ }^{58}$ In clinical studies, miR-146a was also found to be overexpressed in valvular tissue from patients with atherosclerosis, suggesting an association of miR-146a with aortic valve stenosis. ${ }^{59}$ Likewise, Raitoharju et al reported that miR-146a levels were elevated in plaque from aortic, carotid, and femoral atherosclerotic arteries versus nonatherosclerotic left internal thoracic arteries. ${ }^{60}$ Interestingly, a study associated miR-146a levels with plaque stability in coronary stenotic lesions. ${ }^{61}$ The authors found raised miR-146a levels in human peripheral blood mononuclear cells (PBMCs) from vulnerable plaque group versus those from stable plaque group. ${ }^{61}$ Accordingly, miR-146a was also increased in PBMCs samples from CAD patients. ${ }^{62}$ This apparent discrepancy with the animal studies showing that upregulation of miR-146a is atheroprotective might be explained by a compensatory upregulation of miR-146a in response to activation of NF-KB signaling in atherosclerosis, as part of a negative feedback loop. Overall, the role of miR-146a in atherosclerosis appears complex, and it is important to therapeutically focus on a specific cell type at a particular stage of atherogenesis.

Importantly, various association studies have indicated that MIR146A miR-SNPs (rs2431697 and rs2910164) play a role in atherosclerosis-related diseases (i.e., CAD and IS) development and progression. These studies have been performed in different populations and genetic make-up. Our group demonstrated that the T variant of rs2431697 (associated with low miR-146a levels) was predictor of adverse cardiovascular events, such as IS, in a cohort of 901 AF patients. ${ }^{6}$ Subsequently, we evidenced that the TT genotype was associated with high inflammatory status and NET release, which could explain its prothrombotic effect. ${ }^{40}$ In agreement with our findings, Wang et $\mathrm{a}^{63}$ found that rs2431697 T carriers had an increased CAD risk in a Chinese population.

In contrast, the relationship between rs2910164 and CVD has been widely studied although the results are still inconclusive. Zhong et $\mathrm{al}^{64}$ revealed that rs2910164 was associated with an increased risk of atherosclerotic cerebral infarction (ACI) in a Chinese cohort. Patients with reduced miR-146a expression in PBMCs exhibited an increased risk of ACI. Two additional studies evaluated the effect of rs2910164, finding that the $G$ allele was associated with an increased risk of stroke or CAD. ${ }^{65,66}$ Ramkaran et al described higher levels of miR-146a in PBMCs from young CAD patient carrying rs2910164 CC genotype. ${ }^{67}$ These patients displayed significantly lower levels of IRAK1 and TRAF6, together with low 
levels of NF-кB and C-reactive protein. Their observations implicated a protective function of $\mathrm{CC}$ genotype by increasing miR-146a levels and reducing inflammation in CAD patients. ${ }^{67}$ However, other researchers have shown that the $G$ allele of rs2910164 decreased the risk of CAD by downregulating the expression of miR-146a. ${ }^{63,68,69}$ Hence, the inconsistency between results of several publications could be attributed to different ethnic groups and study designs. It is important to note that most of the studies were performed in Chinese populations and data gaps are evident in the Caucasian population. Clearly, further studies, including different geographical domains and larger sample sizes, are needed to globally understand the role of these miR-SNPs in CVD.

\section{Myocardial Infarction}

Several evidences directly link miR-146a and MI pathology. Notably, an altered expression of miR-146a has been found in autopsied heart tissue from MI patients compared with control hearts. ${ }^{70}$ Indeed, MI patients with complications including ventricular rupture (VR) had upregulated tissue miR-146a levels versus those without VR. ${ }^{70}$ The authors suggested that miR-146a increased in response to an intense inflammatory reaction that leads to the pathogenesis of VR after MI. ${ }^{70}$ In addition, the role of miR-146a in heart diseases has been widely studied in animal models. However, it has not yet been determined whether its role is protective or harmful to the heart since miR-146a function varies depending on the heart disease model used. Wang et $\mathrm{al}^{71}$ reported that miR-146a transfection into mouse hearts protected against myocardial I/R injury. miR-146a significantly decreased myocardial infarct size and attenuated myocardial apoptosis through the attenuation of NF-KB activation and inflammatory cytokine production by suppressing Irak 1 and Traf6 expression. ${ }^{71}$ Similarly, another recent study found that the injection of miR-146a-transfected human mesenchymal stem cells (hMSC-miR-146a) after myocardial $\mathrm{I} / \mathrm{R}$ injury improved cardiac function in rats $^{72}$ by reducing the fibrotic area through the secretion of vascular endothelial growth factor. ${ }^{72}$ A new finding indicated that exosomes derived from miR-146a-modified adipose stem cells play a key role in cardioprotection after MI by suppressing MI-induced apoptosis, inflammatory response, and fibrosis in an MI rat model. ${ }^{73}$ Moreover, both in vivo and in vitro experiments found that miR-146a directly targeted early growth response factor 1 , a wellknown inducer of myocardial damage, and reverse MI or hypoxia-induced TLR4-NF-KB signal activation. ${ }^{73}$ Another study in miR-146a $a^{-1-}$ mice revealed that miR146a deficiency increased infarct size and apoptosis after $\mathrm{I} / \mathrm{R}$ injury through the upregulation of 19 apoptosis-related genes such as mediator complex subunit $1(\operatorname{Med} 1){ }^{74}$ On the other hand, tyrosine kinases inhibitors agents such as sunitinib (SNT), beyond their beneficial effects on cancer, have shown adverse effects on the cardiovascular system. ${ }^{75}$ Interestingly, Shen et al showed a significant downregulation of miR-146a in the myocardium of SNT-treated mice. ${ }^{75}$ Indeed, they demonstrated the protective effect of
miR-146a upregulation on SNT-induced cardiac contractile dysfunction in vivo and in vitro by targeting cardiac phospholamban and ankyrin-2, both strongly involved in cardiac contractility. ${ }^{75}$ Lastly, interesting data suggested that combination of miR-21 and miR-146a injection in mice (both with cytoprotective roles) had a greater protective effect against cardiac I/R-induced apoptosis compared with their individual effect. ${ }^{76}$ Thus, Huang et al showed that this synergistic action was mediated by enhanced inhibition of apoptosis of cardiomyocytes by the miR-21/PTEN-AKT/pp38 caspase- 3 and miR-146a/TRAF6/p-p38 caspase- 3 signal pathways. ${ }^{76}$ Nevertheless, despite most studies attribute to miR-146a a cardioprotective role, others opposite them. ${ }^{77-79}$ Particularly, it is worth mentioning the study by Oh et al that tested the effects of miR-146a modulation in transverse aortic constriction-induced heart failure (HF) models. ${ }^{78}$ They demonstrated that overexpression of miR-146a attenuated cardiac contractile function by direct inhibition of the small ubiquitin-like modifier 1 (Sumo1) and the sarcoplasmic reticulum $\mathrm{Ca}^{2+}$-ATPase (SERCA2a) expression, both previously associated with HF protection.

Therefore, further investigation is needed to clearly define the role of miR-146a during HF in particular and CVD in general, and to better define the cell-specific function of miR-146a under different pathological stresses.

\section{Sepsis}

Sepsis is the paradigm for inflammatory systemic diseases associating high morbidity and mortality in intensive care units. ${ }^{80}$ Innate and adaptive immune systems' dysfunctions participate as drivers of this pathology in a network not fully known. Since activation of the coagulation cascade occurs in most patients with sepsis, the interplay between inflammation and coagulation has a crucial role in its pathophysiology. ${ }^{81}$ Within hours after its initiation, the severe systemic inflammatory response shifts to an adaptive anti-inflammatory state caused in part by endotoxin tolerance that plays a key role in sepsis by limiting the negative consequences of an excessive inflammation and the endotoxin shock. ${ }^{82}$ Several studies strongly support a crucial role for miR-146a in endotoxin tolerance by acting as a fine-tuning mechanism to prevent an overstimulation of the inflammatory response to persistent bacteria exposures. ${ }^{47,83-86}$ In vitro experiments using human monocytic cells reported that miR-146a levels increased following LPS treatment (through TLR4) and negatively correlated with inflammatory cytokines as cells develop a status of LPS tolerance. ${ }^{31}$ Importantly, tolerance induction required miR-146a upregulation and transfection of exogenous miR-146a prompted endotoxin tolerance, even in the absence of LPS priming. ${ }^{31}$ Subsequent works confirmed these findings and showed that miR-146a was necessary for LPS-induced cross-tolerance to different TLR ligands. ${ }^{31,87,88}$ Interestingly, miR-146a protection in endotoxin tolerance was also observed in morphine treatment, the main analgesic used in postoperative pain management, and a prevalent recreational drug with well-known adverse effects on the immune system. ${ }^{85}$ Chronic morphine treatment mitigated endotoxin tolerance, resulting in persistent inflammation, septicemia, and septic shock by 
downregulating LPS-induced miR-146a in macrophages. ${ }^{85}$ Complementary studies by using various animal models have confirmed the critical role of miR-146a in the control of inflammation and organ dysfunction during bacteria or endotoxin-induced sepsis, involving macrophages as a major mechanism of innate immunity defense. In 2019, Funahashi et al demonstrated that miR-146a induction in splenic macrophages led to the attenuation of excessive inflammation, mortality rate, and severity of organ injury from polymicrobial sepsis induced by cecal ligation puncture (CLP) in mice. ${ }^{89}$ Another recent work reported the protective role of miR-146a in LPS-induced organ damage and in the inflammatory response in mice by inhibiting the Notch1 signal in macrophages. This finding suggested miR146a-Notch1-NF-кB axis as a potential target for the treatment and prevention of sepsis. ${ }^{90}$ Concordantly, miR-146a $a^{-1-}$ mice succumbed earlier than WT to septic shock induced by LPS. ${ }^{30}$ Pan et al provided evidence supporting Jumonji domain-containing protein D3 (JMJD3), an histone lysine demethylase, as an epigenetic regulator of miR-146a transcription in an Escherichia coli-induced sepsis model. ${ }^{91}$ The authors showed that inhibition of JMJD3, upregulated miR-146a transcription in peritoneal macrophages, protecting mice against early septic death. ${ }^{91}$ Alternatively, Song et al found in a CLP-induced sepsis that miR-146a upregulated by IL-1 $\beta$ was selectively packaged into exosomes, transferred to recipient macrophages, where it regulated M1-M2 transition, and finally led to reduced inflammation and increased survival in septic mice. ${ }^{92}$

miR-146a dysregulation has also been described in sepsis in humans. With an interesting approach, Braza-Boïls et al have described that miR-146a was significantly downregulated in plasma after LPS treatment in an experimental human model of low-dose endotoxemia in volunteers. ${ }^{93}$ These results suggest that LPS alone could also be inducing changes in miR-146a expression in humans. Furthermore, miR-146a dysregulation has also been associated with clinical manifestations of sepsis. The downregulation of miR-146a in PBMCs from septic patients have been correlated with elevated IL-6 and monocyte proliferation. ${ }^{94}$ Indeed, IL-6 levels directly correlated with Sequential Organ Failure Assessment score in these patients. ${ }^{94}$ In addition, a couple of reports showed indirect associations between miR-146a miR-SNPs, its targets and sepsis. Shao et al found for the first time a significant association between rs2910164 genotype (but not with rs57095329), miR-146a levels, and the susceptibility for sepsis, ${ }^{80}$ although they could not demonstrate that miR-146a targets were regulated by this miR-SNP. Probably, clinical heterogeneity of these patients and their treatments masked the relation between miR-146a targets and the inflammatory status of septic patients. ${ }^{80}$ Meanwhile, Han et al, reported that rs2910164 genotype conferred a worse outcome in those patients. ${ }^{95}$

On the other hand, cardiovascular complications are major consequences of sepsis/septic shock and are closely associated with increased morbimortality. Hence, Gao et al demonstrated that overexpression of miR-146a into mice myocardium subjected to CLP-induced sepsis protected them against cardiac dysfunction by markedly reducing the infiltration of macrophages and neutrophils into the myocardium and attenuating inflammatory response in both cardiomyocytes and macrophages via suppression of Nf-kB activity through Irak1 and Traf6 downregulation. ${ }^{96}$ Moreover, a subsequent in vitro study reported that the overexpression of miR-146a mitigated the damage of cardiomyocytes induced by LPS in heart-derived H9C2 myocardial cells through negatively regulating NF- $\mathrm{KB}$ activation and inflammatory cytokine production via targeting the type I receptor protein tyrosine Erbb4. ${ }^{97}$ In addition, Xie et al reported that miR-146a, through regulating $\mathrm{Tlr}-4 / \mathrm{Nf}-\mathrm{kB}$ signaling pathway, improved inflammation and decreased myocardial injury markers in rats treated with LPS. ${ }^{98}$ Thus, all these findings support that miR-146a could be a useful agent for protection against sepsis-induced cardiac dysfunction. In fact, we have demonstrated an association between miR-146a rs2431697 genotype and risk for cardiovascular events in CAP patients. ${ }^{41}$ Thus, among 30 hospitalized patients with cardiovascular events, 29 carried the $\mathrm{T}$ allele (relative risk $=9.61,95 \%$ confidence interval 1.28-72.15). Increased cardiovascular risk remained significant for $\mathrm{T}$ carriers 30 days after hospitalization. ${ }^{41}$ Interestingly, our results pointed to NETosis as a functional way by which miR-146a levels lead to thrombosis in sepsis. Thus, among patients with the highest plasma levels of deoxyribonucleic acid/citrullinated histone $\mathrm{H} 3$ (citH3), those bearing $\mathrm{T}$ allele were threefold more frequent than CC. Furthermore, miR146 $a^{-1-}$ mice injected with LPS presented higher citH3 and thrombin-antithrombin complex levels in plasma than WT and more severe lung injury. Based on these results miR-146a might have a role in immunothrombosis in septic patients. ${ }^{41}$ The relationship between miR-146a levels and NETosis in sepsis evolution is an interesting field that we are exploring.

\section{miR-146a as a Plasma Marker}

\section{Cardiovascular Diseases}

After the discovery of miRNA stability in body fluids in 2008, a plethora of studies have evidenced the presence of a variety of circulating cell-free miRNAs. ${ }^{99}$ The ability of these miRNAs to reflect physiological and pathophysiological conditions as well as their high stability in stored patient samples underlines the potential of these molecules to serve as biomarkers for several diseases. ${ }^{100,101}$ Here, we focus on the potential of circulating miR-146a as a biomarker in thromboinflammatory disorders.

In accord with the protective role of miR-146a in atherosclerosis, Wagner et al reported that plasma levels of miR-146a were slightly downregulated in high-density lipoprotein (HDL) fraction from acute coronary syndrome (ACS) patients compared with healthy subjects. ${ }^{102}$ However, this study has two major limitations: (1) the small sample size (10/group) and (2) that HDL-bound miR-146a fraction was less than $1 \%$ and did not correlate with total plasma levels of miR-146a. On the contrary, Oerlemans et al reported that serum miR-146a levels were significantly increased in those patients who developed an ACS, including those with negative high-sensitive cardiac troponin $\mathrm{T}$ (hs-cTnT) or $<3$ hours of onset of chest pain. In 
addition, circulating miR-146a levels discriminate non-ST elevation MI versus unstable angina. ${ }^{103}$ These results were supported by a study showing that serum miR-146a levels were upregulated in CAD patients compared with controls. ${ }^{104}$ In this study, although there were no differences in miR-146a levels between patients with stable versus unstable angina, miR-146a levels were significantly higher in MI versus stable angina patients. Interestingly, this study also showed that miR-146a was mainly associated with serum HDL. ${ }^{104}$ In the same direction, Quan et al found a positive correlation between plasma miR-146a levels and the severity of coronary heart disease (CHD) measured by Gensini score. Interestingly, among CHD patients, those with subclinical hypothyroidism $(\mathrm{SCH})$ exhibited the highest plasma miR-146a levels that positively correlated with thyroid-stimulating hormone levels, thus highlighting a potential predictive value of plasma miR-146a for CHD among individuals with $\mathrm{SCH} .{ }^{105}$ But the most enlightening study showed that plasma miR-146a levels were increased in MI patients compared with control subjects both, before and after percutaneous coronary intervention (PCI), showing a decrease of miR-146a levels after PCI. ${ }^{106}$ They also found a significant positive correlation of miR-146a with other biomarkers such as N-terminal pro-brain natriuretic peptide and Hs-cTNT both, before and after PCI. ${ }^{106}$ Interestingly, it has been proposed that miRNAs can be released into plasma during plaque rupture, thrombus formation, and myocardial I/R injury (necrosis and apoptosis), thus mirroring the levels found in the artery walls of origin. ${ }^{106}$ Whether the increase in plasma miR-146a levels found in CAD/CHD/AMI patients in those studies may reflect a miR-146a-driven pathogenic or, on the contrary, a compensatory mechanism, has yet to be elucidated.

The alteration in circulating miR-146a levels has also been reported in other cardiovascular clinical entities. Halkein et al showed that levels of exosomal miR-146a were significantly higher in plasma from patients with acute peripartum/postpartum cardiomyopathy than in healthy postpartum controls and patients with dilated cardiomyopathy. ${ }^{107}$ Kin et al examined tissue and plasma miRNAs specifically associated with atherosclerotic abdominal aortic aneurysm (AAA), and found that miR-146a was significantly upregulated in AAA tissue compared with normal aortic wall tissue, while it was significantly downregulated in the plasma of AAA patients compared with the plasma from healthy controls. ${ }^{108}$

\section{Sepsis}

Several studies have remarked the role of miR-146a as a promising prognostic and diagnostic biomarker for sepsis. ${ }^{109}$ Wang et al reported that serum level of miR-146a was significantly reduced in patients suffering from sepsis compared with nonseptic systemic inflammatory response syndrome (SIRS) patients and healthy individuals, suggesting that miR-146a could distinguish sepsis from SIRS caused by other noninfectious diseases. ${ }^{110}$ Subsequently, results from another study also showed higher serum miR-146a levels in nonsepsis SIRS patients compared with sepsis patients, ${ }^{111}$ suggesting that miR-146a may be an optimal diagnostic tools for sepsis. Caserta et al confirmed the decrease of miR-146a in sepsis compared with noninfective SIRS. ${ }^{112}$ Recently, Chen et al showed that high levels of miR-146a were associated with higher sepsis risk, disease severity, and systemic inflammation. ${ }^{113}$

\section{Conclusion and Future Perspectives}

Interaction between thrombosis and inflammation is a central feature of several highly prevalent pathologies. Thus, it is essential to understand the mechanisms and to discover new elements leading to thromboinflammatory processes. This is a key to develop new and effective therapeutic tools to fight thrombosis in several diseases. In this context, current evidences strongly support that miR-146a plays a relevant role in arterial thrombosis in diseases with an important inflammatory background. miR-146a is ubiquitously expressed and it exerts several effects in different cell types ranging from endothelial cells and macrophages in atherosclerosis to leukocytes in autoimmune diseases (-Fig. 4; - Table 1). But many questions remain unanswered on the molecular mechanisms and processes that are controlled by miR-146a. Importantly, miR-146a is involved in NET formation although the precise mechanism is unknown. ${ }^{40,41}$ Whether miR-146a affect other cells such as macrophages ${ }^{114}$ or platelets ${ }^{115}$ or processes that may affect NETosis have to be further searched. In addition, the discovery of new miR-146a targets with a role in thrombosis and inflammation may be determinant and approaches using genomics and transcriptomics in the adequate samples from the adequate patients or animal model may help in this endeavor. In this sense, another vital point is to know if miR-SNPs affecting miR-146a levels also produce neutrophil phenotypic changes as those observed in miR-146a $a^{-1-}$ mice and would explain the association between the presence of these genetic alterations and thrombosis in patients with thromboinflammatory diseases such as those described in the present review.

Another interesting aspect is the use of miR-146a as a biomarker of a thromboinflammatory condition. In this case, there is still work to do concerning the standardization of protocols, and this is extensive to any miRNA. There are many limitations in the studies related with the use of miRNAs as markers of diseases and the best way of extraction and quantification from plasma, serum, other fluids, or EVs. Inconsistencies and variations in results between studies can mostly be attributed to preanalytical variation arising from different protocols as well as different normalization strategies. The use of plasma or serum, the purification technique, batch effects, the anticoagulant used, leukocyte and platelet contamination, hemolysis, sample storage time, and quantification technique are important factors that influence miRNA plasma level variability observed between studies. ${ }^{116-119}$ In addition, to obtain enough statistical power, cohorts have to be well calculated to obtain consistent and reproducible results. How and when the samples are obtained also accounts for the large variability observed between studies. For example, drug treatment in CVD or in sepsis can modify miRNA expression levels and may confound the results as shown in several works from Mayr's laboratory among others. ${ }^{93,116,120}$ Thus, given the potential 

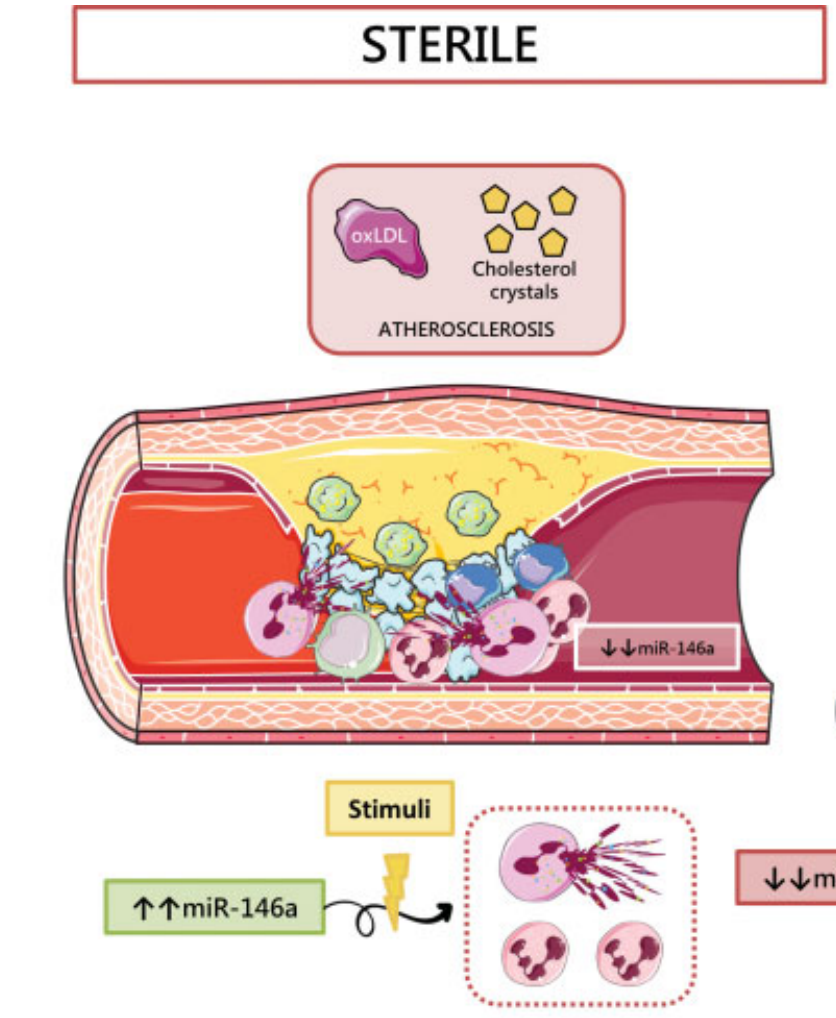

Erythrocyte Foam cell Monocyte $\odot$ Platelet

Neutrophil
Netotic Neutrophil Macrophage

NON-STERILE
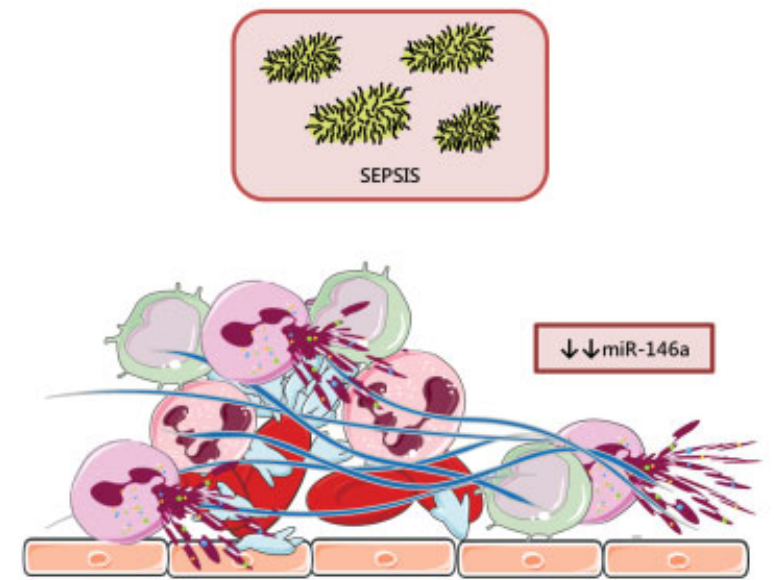

$\downarrow \downarrow$ miR-146a

Stimuli

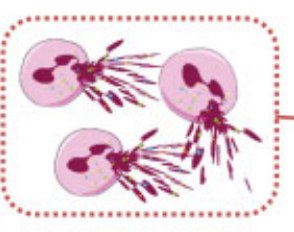

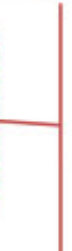

Heart

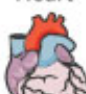

Myocardial

infarction

other CVDs

Lungs

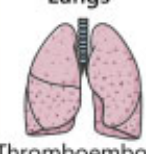

Thromboembolic events

Fig. 4 miR-146a plays a relevant role in thromboinflammation in sterile and nonsterile conditions. The presence of miR-single-nucleotide polymorphisms (SNPs) such as rs2431697 and rs2910164 promote a phenotype with low levels of miR-146a. Upon sterile or nonsterile stimuli, neutrophils expressing the minor alleles would be more prone to NETosis leading to a higher thromboinflammatory process.

Table 1 List of genes targeted by miR-146a

\begin{tabular}{|l|l|l|}
\hline Target gene & Functions & Reference(s) \\
\hline IRAK1 & Essential role in controlling the TLR4/NF-kB pathway & 15 \\
\hline IRAK2 & Component of the IL-1R signaling complex, promotes NF-kB signaling & 123 \\
\hline TRAF6 & Promotes NF-kB signaling & 15 \\
\hline TLR4 & Production of inflammatory cytokines via NF-kB & 28 \\
\hline STAT1 & Crucial role in IFN-y signaling & 45 \\
\hline NOTCH1 & Regulator of macrophage differentiation and activation & 43 \\
\hline NOTCH2 & Development of marginal zone B cells & 124 \\
\hline CXCR4 & Chemokine receptor, involved in calcium mobilization, integrin-mediated & 125 \\
\hline NUMB & adhesion, gene transcription, and proliferation & 124 \\
\hline SOD2 & Negatively regulated NOTCH signaling & 126 \\
\hline EGR1 & Radical scavenger, essential for balancing the intracellular ROS & 73 \\
\hline CARD10 & Promotes TLR4-NF-kB signal activation induced by hypoxia & 127 \\
\hline COPS8 & Specifically required for GPCR-induced NF-kB activation & 127 \\
\hline IL-6, IL-8, CCL5 & Controls NF-kB activation in activated T cells & 128 \\
\hline COX2 & Chemokines for acute inflammatory responses & 129 \\
\hline
\end{tabular}

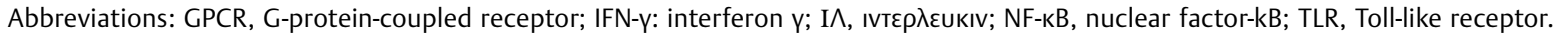


of this technique to diagnose or predict CVD an effort in standardization is necessary to accelerate its use in the future. However, there are already some miRNA panels that are commercially available for the diagnosis of certain pathologies and additional effort must be done to include new applications. ${ }^{121}$

The use of miR-146a as a therapeutic tool is also an exciting future application. Several studies in animal models have shown that miR-146a replacement therapy favors an anti-inflammatory status that could be beneficial in thromboinflammatory diseases such as $\mathrm{CVD}^{122}$ yet no clinical trials have started with miR-146a. Indeed, miRNA therapeutics employing miRNA mimics or antagomirs are currently under clinical trials in different diseases such as cancer, hepatitis C, or HF. ${ }^{122}$ Thus, additional studies on potential off-target effects and efficient delivery methods are needed to allow envisioning miR-146a as an effective therapeutic drug against thromboinflammatory diseases.

\section{Funding}

This work was supported by research grants from Instituto de Salud Carlos III (ISCIII), Fondo Europeo de Desarrollo Regional "Investing in your future" (PI17/00051) (PFIS18/0045: A.M. de los Reyes-García) (CD18/00044: S. Águila) (CM19/00037: L. Reguilón-Gallego), and Fundación Séneca (19873/GERM/15).

\section{Conflict of Interest}

None declared.

\section{Acknowledgments}

The authors would like to acknowledge Servier Medical Art (https://smart.servier.com/; Laboratoires Servier, France) for providing templates that were used for creating figures.

\section{References}

1 Guo L, Rondina MT. The era of thromboinflammation: platelets are dynamic sensors and effector cells during infectious diseases. Front Immunol 2019;10:2204

2 d'Alessandro E, Becker C, Bergmeier W, et al; Scientific Reviewer Committee. Thrombo-inflammation in cardiovascular disease: an expert consensus document from the Third Maastricht Consensus Conference on Thrombosis. Thromb Haemost 2020;120 (04):538-564

3 Saba R, Sorensen DL, Booth SA. MicroRNA-146a: a dominant, negative regulator of the innate immune response. Front Immunol 2014;5:578

4 Paterson MR, Kriegel AJ. MiR-146a/b: a family with shared seeds and different roots. Physiol Genomics 2017;49(04):243-252

5 Zhang Z, Zhang Y, Sun XX, Ma X, Chen ZN. microRNA-146a inhibits cancer metastasis by downregulating VEGF through dual pathways in hepatocellular carcinoma. Mol Cancer 2015; 14:5

6 Roldán V, Arroyo AB, Salloum-Asfar S, et al. Prognostic role of MIR146A polymorphisms for cardiovascular events in atrial fibrillation. Thromb Haemost 2014;112(04):781-788

7 Marschner D, Falk M, Javorniczky NR, et al. MicroRNA-146a regulates immune-related adverse events caused by immune checkpoint inhibitors. JCI Insight 2020;5(06):e132334
8 Nakada TA, Takahashi W, Nakada E, Shimada T, Russell JA, Walley KR. Genetic polymorphisms in sepsis and cardiovascular disease: do similar risk genes suggest similar drug targets? Chest 2019; 155(06):1260-1271

9 Montecucco F, Liberale L, Bonaventura A, Vecchiè A, Dallegri F, Carbone F. The role of inflammation in cardiovascular outcome. Curr Atheroscler Rep 2017;19(03):11

10 Ayoub KF, Pothineni NVK, Rutland J, Ding Z, Mehta JL. Immunity, inflammation, and oxidative stress in heart failure: emerging molecular targets. Cardiovasc Drugs Ther 2017;31(5-6):593-608

11 Calin GA, Dumitru CD, Shimizu M, et al. Frequent deletions and down-regulation of micro- RNA genes miR15 and miR16 at $13 q 14$ in chronic lymphocytic leukemia. Proc Natl Acad Sci U S A 2002;99(24):15524-15529

12 Bartel DP. MicroRNAs: target recognition and regulatory functions. Cell 2009;136(02):215-233

13 Hendrickson DG, Hogan DJ, McCullough HL, et al. Concordant regulation of translation and mRNA abundance for hundreds of targets of a human microRNA. PLoS Biol 2009;7(11):e1000238

14 Bartel DP. Metazoan microRNAs. Cell 2018;173(01):20-51

15 Taganov KD, Boldin MP, Chang KJ, Baltimore D. NF-kappaBdependent induction of microRNA miR-146, an inhibitor targeted to signaling proteins of innate immune responses. Proc Natl Acad Sci U S A 2006;103(33):12481-12486

16 Curtale G, Citarella F, Carissimi C, et al. An emerging player in the adaptive immune response: microRNA-146a is a modulator of IL-2 expression and activation-induced cell death in T lymphocytes. Blood 2010;115(02):265-273

17 Luo X, Yang W, Ye DQ et al. A functional variant in microRNA146a promoter modulates its expression and confers disease risk for systemic lupus erythematosus. PLoS Genet 2011;7(06): e1002128

18 Chang TC, Yu D, Lee YS, et al. Widespread microRNA repression by Myc contributes to tumorigenesis. Nat Genet 2008;40(01): 43-50

19 Jazdzewski K, Murray EL, Franssila K, Jarzab B, Schoenberg DR, de la Chapelle A. Common SNP in pre-miR-146a decreases mature miR expression and predisposes to papillary thyroid carcinoma. Proc Natl Acad Sci U S A 2008;105(20):7269-7274

20 Löfgren SE, Frostegård J, Truedsson L, et al. Genetic association of miRNA-146a with systemic lupus erythematosus in Europeans through decreased expression of the gene. Genes Immun 2012; 13(03):268-274

21 Stickel N, Hanke K, Marschner D, et al. MicroRNA-146a reduces MHC-II expression via targeting JAK/STAT signaling in dendritic cells after stem cell transplantation. Leukemia 2017;31(12): 2732-2741

22 Bastami M, Choupani J, Saadatian Z, et al. MiRNA polymorphisms and risk of cardio-cerebrovascular diseases: a systematic review and meta-analysis. Int J Mol Sci 2019;20(02):293

23 Ding Y, Guo F, Zhu T, et al. Mechanism of long non-coding RNA MALAT1 in lipopolysaccharide-induced acute kidney injury is mediated by the miR-146a/NF-kB signaling pathway. Int J Mol Med 2018;41(01):446-454

24 Dai L, Zhang G, Cheng Z, et al. Knockdown of LncRNA MALAT1 contributes to the suppression of inflammatory responses by upregulating miR-146a in LPS-induced acute lung injury. Connect Tissue Res 2018;59(06):581-592

25 Sun W, Ma M, Yu H, Yu H. Inhibition of IncRNA X inactivatespecific transcript ameliorates inflammatory pain by suppressing satellite glial cell activation and inflammation by acting as a sponge of miR-146a to inhibit $\mathrm{Na}_{\mathrm{v}}$ 1.7. J Cell Biochem 2018;119 (12):9888-9898

26 Zhou Y-X, Zhao W, Mao L-W, et al. Long non-coding RNA NIFKAS1 inhibits M2 polarization of macrophages in endometrial cancer through targeting miR-146a. Int J Biochem Cell Biol 2018; 104:25-33 
27 Liu W, Wu YH, Zhang L, et al. MicroRNA-146a suppresses rheumatoid arthritis fibroblast-like synoviocytes proliferation and inflammatory responses by inhibiting the TLR4/NF-kB signaling. Oncotarget 2018;9(35):23944-23959

28 Yang K, He YS, Wang XQ et al. MiR-146a inhibits oxidized low-density lipoprotein-induced lipid accumulation and inflammatory response via targeting toll-like receptor 4. FEBS Lett 2011;585(06):854-860

29 Ye EA, Steinle JJEA YmiR-146a attenuates inflammatory pathways mediated by TLR4/NF-KB and TNF $\alpha$ to protect primary human retinal microvascular endothelial cells grown in high glucose. Mediators Inflamm 2016;2016:3958453

30 Boldin MP, Taganov KD, Rao DS, et al. miR-146a is a significant brake on autoimmunity, myeloproliferation, and cancer in mice. J Exp Med 2011;208(06):1189-1201

31 Nahid MA, Pauley KM, Satoh M, Chan EKL. miR-146a is critical for endotoxin-induced tolerance: implication in innate immunity. J Biol Chem 2009;284(50):34590-34599

32 Zhao JL, Rao DS, Boldin MP, Taganov KD, O'Connell RM, Baltimore D. NF-kappaB dysregulation in microRNA-146a-deficient mice drives the development of myeloid malignancies. Proc Natl Acad Sci U S A 2011;108(22):9184-9189

33 Ferrer-Marín F, Arroyo AB, Bellosillo B, et al; GEMFIN Group. miR146a rs2431697 identifies myeloproliferative neoplasm patients with higher secondary myelofibrosis progression risk. Leukemia 2020;34(10):2648-2659

34 Zhao JL, Rao DS, O'Connell RM, Garcia-Flores Y, Baltimore D. MicroRNA-146a acts as a guardian of the quality and longevity of hematopoietic stem cells in mice. eLife 2013;2:e00537

35 Liew PX, Kubes P. The neutrophil's role during health and disease. Physiol Rev 2019;99(02):1223-1248

36 Brinkmann V, Reichard U, Goosmann C, et al. Neutrophil extracellular traps kill bacteria. Science 2004;303(5663):1532-1535

37 Papayannopoulos V. Neutrophil extracellular traps in immunity and disease. Nat Rev Immunol 2018;18(02):134-147

38 Camicia G, Pozner R, de Larrañaga G. Neutrophil extracellular traps in sepsis. Shock 2014;42(04):286-294

39 Laridan E, Martinod K, De Meyer SF. Neutrophil extracellular traps in arterial and venous thrombosis. Semin Thromb Hemost 2019;45(01):86-93

40 Arroyo AB, de Los Reyes-García AM, Rivera-Caravaca JM, et al. MiR-146a regulates neutrophil extracellular trap formation that predicts adverse cardiovascular events in patients with atrial fibrillation. Arterioscler Thromb Vasc Biol 2018;38(04):892-902

41 Arroyo AB, Fernández-Pérez MP, Del Monte A, et al. miR-146a is a pivotal regulator of neutrophil extracellular trap formation promoting thrombosis. Haematologica 2020:haematol. 2019.240226-haematol.2019.240226

42 Yang S, Yuan HQ Hao YM, et al. Macrophage polarization in atherosclerosis. Clin Chim Acta 2020;501:142-146

43 Huang C, Liu XJ, Qun Zhou, et al. MiR-146a modulates macrophage polarization by inhibiting Notch1 pathway in RAW264.7 macrophages. Int Immunopharmacol 2016;32:46-54

44 Curtale G, Rubino M, Locati M. MicroRNAs as molecular switches in macrophage activation. Front Immunol 2019;10:799

45 He X, Tang R, Sun Y, et al. MicroR-146 blocks the activation of M1 macrophage by targeting signal transducer and activator of transcription 1 in hepatic schistosomiasis. EBioMedicine 2016;13:339-347

46 Li Z, Wang S, Zhao W, Sun Z, Yan H, Zhu J. Oxidized low-density lipoprotein upregulates microRNA-146a via JNK and NF-кB signaling. Mol Med Rep 2016;13(02):1709-1716

47 Nahid MA, Benso LM, Shin JD, Mehmet H, Hicks A, Ramadas RA. TLR4, TLR7/8 agonist-induced miR-146a promotes macrophage tolerance to MyD88-dependent TLR agonists. J Leukoc Biol 2016; 100(02):339-349

48 Dai Y, Jia P, Fang Y, et al. miR-146a is essential for lipopolysaccharide (LPS)-induced cross-tolerance against kidney ischemia/ reperfusion injury in mice. Sci Rep 2016;6:27091
49 Yang L, Boldin MP, Yu Y, et al. miR-146a controls the resolution of T cell responses in mice. J Exp Med 2012;209(09):1655-1670

50 Magilnick N, Reyes EY, Wang WL, et al. miR-146a-Traf6 regulatory axis controls autoimmunity and myelopoiesis, but is dispensable for hematopoietic stem cell homeostasis and tumor suppression. Proc Natl Acad Sci U S A 2017;114(34):E7140-E7149

51 Fish JE, Cybulsky MI, Apo E. ApoE attenuates atherosclerosis via miR-146a. Circ Res 2015;117(01):3-6

52 Li K, Ching D, Luk FS, Raffai RL. Apolipoprotein E enhances microRNA-146a in monocytes and macrophages to suppress nuclear factor-KB-driven inflammation and atherosclerosis. Circ Res 2015;117(01):e1-e11

53 Del Monte A, Arroyo AB, Andrés-Manzano MJ, et al. miR-146a deficiency in hematopoietic cells is not involved in the development of atherosclerosis. PLoS One 2018;13(06):e0198932

54 Cheng HS, Besla R, Li A, et al. Paradoxical suppression of atherosclerosis in the absence of microRNA-146a. Circ Res 2017;121(04):354-367

55 Ma S, Tian XY, Zhang Y, et al. E-selectin-targeting delivery of microRNAs by microparticles ameliorates endothelial inflammation and atherosclerosis. Sci Rep 2016;6:22910

56 Gareus R, Kotsaki E, Xanthoulea S, et al. Endothelial cell-specific NF-kappaB inhibition protects mice from atherosclerosis. Cell Metab 2008;8(05):372-383

57 Kanters E, Pasparakis M, Gijbels MJJ, et al. Inhibition of NF-kappaB activation in macrophages increases atherosclerosis in LDL receptor-deficient mice. J Clin Invest 2003;112(08):1176-1185

58 Nguyen MA, Karunakaran D, Geoffrion M, et al. Extracellular vesicles secreted by atherogenic macrophages transfer microRNA to inhibit cell migration. Arterioscler Thromb Vasc Biol 2018;38(01):49-63

59 Petrkova J, Borucka J, Kalab M, et al. Increased expression of miR$146 \mathrm{a}$ in valvular tissue from patients with aortic valve stenosis. Front Cardiovasc Med 2019;6:86

60 Raitoharju E, Lyytikäinen LP, Levula M, et al. miR-21, miR-210, miR-34a, and miR-146a/b are up-regulated in human atherosclerotic plaques in the Tampere Vascular Study. Atherosclerosis 2011;219(01):211-217

61 Zhu GF, Chu T, Ruan Z, et al. Inflammation-related MicroRNAs are associated with plaque stability calculated by IVUS in coronary heart disease patients. J Interv Cardiol 2019;2019:9723129

62 Takahashi Y, Satoh M, Minami Y, Tabuchi T, Itoh T, Nakamura M. Expression of miR-146a/b is associated with the Toll-like receptor 4 signal in coronary artery disease: effect of reninangiotensin system blockade and statins on miRNA-146a/b and Toll-like receptor 4 levels. Clin Sci (Lond) 2010;119(09): 395-405

63 Wang Y, Wang X, Li Z, et al. Two single nucleotide polymorphisms (rs2431697 and rs2910164) of miR-146a are associated with risk of coronary artery disease. Int J Environ Res Public Health 2017;14(05):4-10

64 Zhong H, Cai Y, Cheng J, et al. Apolipoprotein E epsilon 4 enhances the association between the rs2910164 polymorphism of miR-146a and risk of atherosclerotic cerebral infarction. J Atheroscler Thromb 2016;23(07):819-829

65 Jeon YJ, Kim OJ, Kim SY, et al. Association of the miR-146a, miR149, miR-196a2, and miR-499 polymorphisms with ischemic stroke and silent brain infarction risk. Arterioscler Thromb Vasc Biol 2013;33(02):420-430

66 Sung J-H, Kim S-H, Yang W-I, et al. miRNA polymorphisms (miR-146a, miR-149, miR-196a2 and miR-499) are associated with the risk of coronary artery disease. Mol Med Rep 2016;14 (03):2328-2342

67 Ramkaran P, Khan S, Phulukdaree A, Moodley D, Chuturgoon AA. miR-146a polymorphism influences levels of miR-146a, IRAK-1, and TRAF- 6 in young patients with coronary artery disease. Cell Biochem Biophys 2014;68(02):259-266 
68 Xiong X, Cho M, Cai X, Cheng J, Jing X. Mutation research / fundamental and molecular mechanisms of mutagenesis: a common variant in pre-miR-146 is associated with coronary artery disease risk and its mature miRNA expression. Mutat Res Fundam Mol Mech Mutagen 2014;•••:76115-76120

69 Bastami M, Ghaderian SMH, Omrani MD, et al. MiRNA-related polymorphisms in miR-146a and TCF21 are associated with increased susceptibility to coronary artery disease in an Iranian population. Genet Test Mol Biomarkers 2016;20(05):241-248

70 Zidar N, Boštjančič E, Glavač D, Štajer D. MicroRNAs, innate immunity and ventricular rupture in human myocardial infarction. Dis Markers 2011;31(05):259-265

71 Wang X, Ha T, Liu L, et al. Increased expression of microRNA146a decreases myocardial ischaemia/reperfusion injury. Cardiovasc Res 2013;97(03):432-442

72 Seo HH, Lee SY, Lee CY, et al. Exogenous miRNA-146a enhances the therapeutic efficacy of human mesenchymal stem cells by increasing vascular endothelial growth factor secretion in the ischemia/ reperfusion-injured heart. J Vasc Res 2017;54(02):100-108

73 Pan J, Alimujiang M, Chen Q Shi H, Luo X. Exosomes derived from miR-146a-modified adipose-derived stem cells attenuate acute myocardial infarction-induced myocardial damage via downregulation of early growth response factor 1 . J Cell Biochem 2019;120(03):4433-4443

74 Zhang T, Ma Y, Gao L, et al. MicroRNA-146a protects against myocardial ischaemia reperfusion injury by targeting Med1. Cell Mol Biol Lett 2019;24:62

75 Shen L, Li C, Zhang H, Qiu S, Fu T, Xu Y. Downregulation of miR146 a contributes to cardiac dysfunction induced by the tyrosine kinase inhibitor sunitinib. Front Pharmacol 2019;10:914

76 Huang W, Tian SS, Hang PZ, Sun C, Guo J, Du ZM. Combination of microRNA-21 and microRNA-146a attenuates cardiac dysfunction and apoptosis during acute myocardial infarction in mice. Mol Ther Nucleic Acids 2016;5:e296

77 He J, Lu Y, Song X, Gong X, Li Y. Inhibition of microRNA-146a attenuated heart failure in myocardial infarction rats. Biosci Rep 2019;39(12):1-14

78 Oh JG, Watanabe S, Lee A, et al. miR-146a suppresses SUMO1 expression and induces cardiac dysfunction in maladaptive hypertrophy. Circ Res 2018;123(06):673-685

79 Shu L, Zhang W, Huang G, et al. Troxerutin attenuates myocardial cell apoptosis following myocardial ischemia-reperfusion injury through inhibition of miR-146a-5p expression. J Cell Physiol 2019;234(06):9274-9282

80 Shao Y, Li J, Cai Y, et al. The functional polymorphisms of miR146a are associated with susceptibility to severe sepsis in the Chinese population. Mediators Inflamm 2014;2014:916202

81 Iba T, Levy JH. Inflammation and thrombosis: roles of neutrophils, platelets and endothelial cells and their interactions in thrombus formation during sepsis. J Thromb Haemost 2018;16 (02):231-241

82 Bosmann M, Ward PA. The inflammatory response in sepsis. Trends Immunol 2013;34(03):129-136

83 Brudecki L, Ferguson DA, McCall CE, El Gazzar M. MicroRNA146a and RBM4 form a negative feed-forward loop that disrupts cytokine mRNA translation following TLR4 responses in human THP-1 monocytes. Immunol Cell Biol 2013;91(08):532-540

84 Testa U, Pelosi E, Castelli G, Labbaye C. miR-146 and miR-155: two key modulators of immune response and tumor development. Noncoding RNA 2017;3(03):22

85 Banerjee S, Meng J, Das S, et al. Morphine induced exacerbation of sepsis is mediated by tempering endotoxin tolerance through modulation of miR-146a. Sci Rep 2013;3:1977

86 Nahid MA, Satoh M, Chan EKL. Interleukin 1 $\beta$-responsive microRNA-146a is critical for the cytokine-induced tolerance and cross-tolerance to Toll-like receptor ligands. J Innate Immun 2015;7(04):428-440
87 Liu M, John CM, Jarvis GA. Induction of endotoxin tolerance by pathogenic Neisseria is correlated with the inflammatory potential of lipooligosaccharides and regulated by microRNA-146a. J Immunol 2014;192(04):1768-1777

88 Molteni M, Bosi A, Saturni V, Rossetti C. MiR-146a induction by cyanobacterial lipopolysaccharide antagonist (CyP) mediates endotoxin cross-tolerance. Sci Rep 2018;8(01):11367

89 Funahashi Y, Kato N, Masuda T, et al. miR-146a targeted to splenic macrophages prevents sepsis-induced multiple organ injury. Lab Invest 2019;99(08):1130-1142

90 Bai X, Zhang J, Cao M, et al. MicroRNA-146a protects against LPSinduced organ damage by inhibiting Notch1 in macrophage. Int Immunopharmacol 2018;63:220-226

91 Pan Y, Wang J, Xue Y, et al. GSKJ4 protects mice against early sepsis via reducing proinflammatory factors and up-regulating MiR-146a. Front Immunol 2018;9:2272

92 Song Y, Dou H, Li X, et al. Exosomal miR-146a contributes to the enhanced therapeutic efficacy of interleukin-1 $\beta$-primed mesenchymal stem cells against sepsis. Stem Cells 2017;35(05):1208-1221

93 Braza-Boïls A, Barwari T, Gutmann C, et al. Circulating microRNA levels indicate platelet and leukocyte activation in endotoxemia despite platelet p2y12 inhibition. Int J Mol Sci 2020;21(08):2897

94 Zhou J, Chaudhry H, Zhong Y, et al. Dysregulation in microRNA expression in peripheral blood mononuclear cells of sepsis patients is associated with immunopathology. Cytokine 2015; 71(01):89-100

95 Han Y, Li Y, Jiang Y. The prognostic value of plasma microRNA-155 and microRNA-146a level in severe sepsis and sepsis-induced acute lung injury patients. Clin Lab 2016;62(12):2355-2360

96 Gao M, Wang X, Zhang X, et al. Attenuation of cardiac dysfunction in polymicrobial sepsis by microRNA-146a is mediated via targeting of IRAK1 and TRAF6 expression. J Immunol 2015;195 (02):672-682

97 An R, Feng J, Xi C, Xu J, Sun L. miR-146a attenuates sepsis-induced myocardial dysfunction by suppressing IRAK1 and TRAF6 via targeting ErbB4 expression. Oxid Med Cell Longev 2018; 2018:7163057

98 Xie J, Zhang L, Fan X, Dong X, Zhang Z, Fan W. MicroRNA-146a improves sepsis-induced cardiomyopathy by regulating the TLR4/NF-kB signaling pathway. Exp Ther Med 2019;18(01):779-785

99 Mitchell PS, Parkin RK, Kroh EM, et al. Circulating microRNAs as stable blood-based markers for cancer detection. Proc Natl Acad Sci U S A 2008;105(30):10513-10518

100 Grasedieck S, Sorrentino A, Langer C, et al. Circulating microRNAs in hematological diseases: principles, challenges, and perspectives. Blood 2013;121(25):4977-4984

101 Halushka PV, Goodwin AJ, Halushka MK. Opportunities for microRNAs in the crowded field of cardiovascular biomarkers. Annu Rev Pathol 2019;14:211-238

102 Wagner J, Riwanto M, Besler C, et al. Characterization of levels and cellular transfer of circulating lipoprotein-bound microRNAs. Arterioscler Thromb Vasc Biol 2013;33(06):1392-1400

103 Oerlemans MIFJ, Mosterd A, Dekker MS, et al. Early assessment of acute coronary syndromes in the emergency department: the potential diagnostic value of circulating microRNAs. EMBO Mol Med 2012;4(11):1176-1185

104 Niculescu LS, Simionescu N, Sanda GM, et al. MiR-486 and miR92a identified in circulating HDL discriminate between stable and vulnerable coronary artery disease patients. PLoS One 2015; 10(10):e0140958

105 Quan X, Ji Y, Zhang C, et al. Circulating MiR-146a may be a potential biomarker of coronary heart disease in patients with subclinical hypothyroidism. Cell Physiol Biochem 2018;45(01): 226-236

106 Xue S, Zhu W, Liu D, et al. Circulating miR-26a-1, miR-146a and miR-199a-1 are potential candidate biomarkers for acute myocardial infarction. Mol Med 2019;25(01):18 
107 Halkein J, Tabruyn SP, Ricke-Hoch M, et al. MicroRNA-146a is a therapeutic target and biomarker for peripartum cardiomyopathy. J Clin Invest 2013;123(05):2143-2154

108 Kin K, Miyagawa S, Fukushima S, et al. Tissue- and plasmaspecific MicroRNA signatures for atherosclerotic abdominal aortic aneurysm. J Am Heart Assoc 2012;1(05):e000745

109 Essandoh K, Fan G-C. Role of extracellular and intracellular microRNAs in sepsis. Biochim Biophys Acta 2014;1842(11): 2155-2162

110 Wang J-F, Yu M-L, Yu G, et al. Serum miR-146a and miR-223 as potential new biomarkers for sepsis. Biochem Biophys Res Commun 2010;394(01):184-188

111 Wang L, Wang HC, Chen C, et al. Differential expression of plasma miR-146a in sepsis patients compared with non-sepsis-SIRS patients. Exp Ther Med 2013;5(04):1101-1104

112 Caserta S, Kern F, Cohen J, Drage S, Newbury SF, Llewelyn MJ. Circulating plasma microRNAs can differentiate human sepsis and systemic inflammatory response syndrome (SIRS). Sci Rep 2016;6:28006

113 Chen L, Yu L, Zhang R, Zhu L, Shen W. Correlation of microRNA$146 \mathrm{a} / \mathrm{b}$ with disease risk, biochemical indices, inflammatory cytokines, overall disease severity, and prognosis of sepsis. Medicine (Baltimore) 2020;99(22):e19754

114 Warnatsch A, Ioannou M, Wang Q, Papayannopoulos V. Inflammation. Neutrophil extracellular traps license macrophages for cytokine production in atherosclerosis. Science 2015;349 (6245):316-320

115 Clark SR, Ma AC, Tavener SA, et al. Platelet TLR4 activates neutrophil extracellular traps to ensnare bacteria in septic blood. Nat Med 2007;13(04):463-469

116 Sunderland N, Skroblin P, Barwari T, et al. MicroRNA biomarkers and platelet reactivity: the clot thickens. Circ Res 2017;120(02): 418-435

117 Ramón-Núñez LA, Martos L, Fernández-Pardo Á, et al. Comparison of protocols and RNA carriers for plasma miRNA isolation. Unraveling RNA carrier influence on miRNA isolation. PLoS One 2017;12(10):e0187005

118 Mompeón A, Ortega-Paz L, Vidal-Gómez X, et al. Disparate miRNA expression in serum and plasma of patients with acute myocardial infarction: a systematic and paired comparative analysis. Sci Rep 2020;10(01):5373

119 Keller A, Rounge T, Backes C, et al. Sources to variability in circulating human miRNA signatures. RNA Biol 2017;14(12): 1791-1798

120 Chyrchel B, Totoń-Żurańska J, Kruszelnicka O, et al. Association of plasma miR-223 and platelet reactivity in patients with coronary artery disease on dual antiplatelet therapy: a preliminary report. Platelets 2015;26(06):593-597

121 Bonneau E, Neveu B, Kostantin E, Tsongalis GJ, De Guire V. How close are miRNAs from clinical practice? A perspective on the diagnostic and therapeutic market. EJIFCC 2019;30(02):114-127

122 Huang CK, Kafert-Kasting S, Thum T. Preclinical and clinical development of noncoding RNA therapeutics for cardiovascular disease. Circ Res 2020;126(05):663-678

123 Hou J, Wang P, Lin L, et al. MicroRNA-146a feedback inhibits RIGI-dependent type I IFN production in macrophages by targeting TRAF6, IRAK1, and IRAK2. J Immunol 2009;183(03):2150-2158

124 King JK, Ung NM, Paing MH, et al. Regulation of marginal zone Bcell differentiation by microRNA-146a. Front Immunol 2017; 7:670

125 Liu W, He J, Yang Y, Guo Q, Gao F. Upregulating miR-146a by physcion reverses multidrug resistance in human chronic myelogenous leukemia K562/ADM cells. Am J Cancer Res 2016;6(11): 2547-2560

126 Ji G, Lv K, Chen H, et al. MiR-146a regulates SOD2 expression in H2O2 stimulated PC12 cells. PLoS One 2013;8(07):e69351

127 Crone SG, Jacobsen A, Federspiel B, et al. microRNA-146a inhibits $\mathrm{G}$ protein-coupled receptor-mediated activation of NF-кB by targeting CARD10 and COPS8 in gastric cancer. Mol Cancer 2012;11:71

128 Perry MM, Moschos SA, Williams AE, Shepherd NJ, LarnerSvensson HM, Lindsay MA. Rapid changes in microRNA-146a expression negatively regulate the IL- $1 \beta$-induced inflammatory response in human lung alveolar epithelial cells. J Immunol 2008;180(08):5689-5698

129 Cornett AL, Lutz CS. Regulation of COX-2 expression by miR-146a in lung cancer cells. RNA 2014;20(09):1419-1430 